DR. GIUSEPPE LIBERATORE (Orcid ID : 0000-0003-2666-1678)

PROF. FIORE MANGANELLI (Orcid ID : 0000-0001-9478-3744)

DR. DARIO COCITO (Orcid ID : 0000-0002-6964-618X)

PROF. CHIARA BRIANI (Orcid ID : 0000-0001-8035-0200)

MISS MASSIMILIANO FILOSTO (Orcid ID : 0000-0002-2852-7512)

DR. L. BENEDETTI (Orcid ID : 0000-0002-9540-9727)

DR. MARCO LUIGETTI (Orcid ID : 0000-0001-7539-505X)

PROF. GIUSEPPE LAURIA (Orcid ID : 0000-0001-9773-020X)

DR. LUCA LEONARDI (Orcid ID : 0000-0002-1267-864X)

DR. EDUARDO NOBILE-ORAZIO (Orcid ID : 0000-0003-2624-8138)

Article type : Original Article

\title{
CHRONIC INFLAMMATORY DEMYELINATING POLYRADICULONEUROPATHY: CAN WE MAKE A DIAGNOSIS IN PATIENTS NOT FULFILLING ELECTRODIAGNOSTIC CRITERIA?
}

Giuseppe Liberatore, ${ }^{1}$ Fiore Manganelli, ${ }^{2}$ Pietro Emiliano Doneddu, ${ }^{1}$ Dario Cocito, ${ }^{3}$ Raffaella Fazio, ${ }_{4}^{4}$ Chiara Briani, ${ }^{5}$ Massimiliano Filosto, ${ }^{6}$ Luana Benedetti, ${ }^{7,8}$ Anna Mazzeo, ${ }^{9}$ Giovanni Antonini, ${ }^{10}$ Giuseppe Cosentino, ${ }^{11,12}$ Stefano Jann, ${ }^{13}$ Andrea Cortese, ${ }^{12,14}$ Girolama Alessandra Marfia, ${ }^{15}$ Angelo Maurizio Clerici, ${ }^{16}$ Gabriele Siciliano, ${ }^{17}$ Marinella Carpo, ${ }^{18}$ Marco Luigetti, ${ }^{19}$ Giuseppe Lauria, ${ }^{20,21}$ Tiziana Rosso, ${ }^{22}$ Guido Cavaletti, ${ }^{23}$ Lucio Santoro, ${ }^{2}$ Erdita Peci, ${ }^{3}$ Stefano This article has been accepted for publication and undergone full peer review but has not been through the copyediting, typesetting, pagination and proofreading process, which may lead to differences between this version and the Version of Record. Please cite this article as doi: 10.1111/ENE.14545

This article is protected by copyright. All rights reserved 
Tronci, ${ }^{4}$ Marta Ruiz, ${ }^{5}$ Stefano Cotti Piccinelli, ${ }^{6}$ Angelo Schenone, ${ }^{7}$ Luca Leonardi, ${ }^{9}$ Antonio Toscano, ${ }^{12}$ Giorgia Mataluni, ${ }^{14}$ Emanuele Spina, ${ }^{2}$ Luca Gentile, ${ }^{9}$ Eduardo Nobile-Orazio, ${ }^{1,24}$ on the behalf of the Italian CIDP Database Study Group.

1. Neuromuscular and Neuroimmunology Service, IRCCS Humanitas Clinical and Research Institute, Rozzano, Milan, Italy

2. Department of Neuroscience, Reproductive Sciences and Odontostomatology, University of Naples 'Federico II', Naples, Italy

3. Presidio Sanitario Major, Istituti Clinici Scientifici Maugeri, Turin, Italy

4. Division of Neuroscience, Department of Neurology, Institute of Experimental Neurology (INSPE), San Raffaele Scientific Institute, Milan, Italy

5. Neurology Unit, Department of Neuroscience, University of Padova, Padova, Italy

6. Center for Neuromuscular Diseases and Neuropathies, Unit of Neurology, ASST 'Spedali Civili', University of Brescia, Brescia, Italy

7. Department of Neuroscience, Rehabilitation, Ophthalmology, Genetics, Maternal and Child Health, University of Genoa and IRCCS AOU San Martino-IST, Genoa, Italy

8. Neurology Unit, Sant'Andrea Hospital, La Spezia, Italy

9. Department of Clinical and Experimental Medicine, Unit of Neurology, University of Messina, Messina, Italy

10. Unit of Neuromuscular Diseases, Department of Neurology Mental Health and Sensory Organs (NESMOS), Faculty of Medicine and Psychology, 'Sapienza' University of Rome, Sant'Andrea Hospital, Rome, Italy

11. Department of Experimental BioMedicine and Clinical Neurosciences (BioNeC), University of Palermo, Palermo, Italy

12. IRCCS Foundation C. Mondino National Neurological Institute, Pavia, Italy

13. Department of Neuroscience, Niguarda Ca' Granda Hospital, Milan, Italy

14. Molecular Neurosciences, University College London, London, UK

15. Dysimmune Neuropathies Unit, Department of Systems Medicine, Tor Vergata University of Rome, Rome, Italy

16. Neurology Unit, Circolo \& Macchi Foundation Hospital, Insubria University, DBSV, Varese, Italy 
17. Neurology Unit, Department of Clinical and Experimental Medicine, University of Pisa, Pisa, Italy

18. Neurology Unit, ASST Bergamo Ovest-Ospedale Treviglio, Treviglio, Italy

19. Fondazione Policlinico Universitario A. Gemelli IRCCS, UOC Neurologia, Università Cattolica del Sacro Cuore, Rome, Italy.

20. Unit of Neuroalgology, IRCCS Foundation 'Carlo Besta' Neurological Institute, Milan, Italy

21. Department of Biomedical and Clinical Sciences 'Luigi Sacco', University of Milan, Milan, Italy

22. ULSS2 Marca Trevigiana, UOC Neurologia-Castelfranco Veneto, Treviso, Italy

23. School of Medicine and Surgery and Experimental Neurology Unit, University of MilanoBicocca, Monza, Italy

24. Department of Medical Biotechnology and Translational Medicine, Milan University, Milan, Italy

Address Correspondence to: Eduardo Nobile-Orazio, MD, PhD, FAAN, FEAN, Neuromuscular and Neuroimmunology Service, IRCCS Humanitas Research Hospital, Via Manzoni 56, Rozzano,

Milan 20089, Italy. Tel: +390282242209; Fax: +390282242298; E-mail:

eduardo.nobile@unimi.it

Submission Type: Original article

Running title: CIDP diagnosis beyond electrophysiology

Number of Tables: $\mathbf{2}$

Number of Supplementary Tables: 1

Number of Figures: 1

Word count of Abstract: 249

Word Count of Paper: 3517

Search Terms: Chronic inflammatory demyelinating polyradiculoneuropathy; CIDP; Diagnostic criteria, Electrophysiology

This article is protected by copyright. All rights reserved 


\section{ABSTRACT}

Objective: to identify the clinical and diagnostic investigations that may help supporting a diagnosis of chronic inflammatory demyelinating polyradiculoneuropathy (CIDP) in patients not fulfilling the EFNS/PNS electrodiagnostic criteria.

Methods: we retrospectively reviewed the data from patients with a clinical diagnosis of CIDP included in a national database.

Results: we included 535 patients with a diagnosis of CIDP. This diagnosis fulfilled the EFNS/PNS criteria in 468 patients (87.2\%) (definite in 430, probable in 33, possible in 3, while two had CISP). Sixty-seven patients had a medical history and clinical signs compatible with CIDP but electrodiagnostic studies did not fulfill the EFNS/PNS criteria for CIDP. These patients had similar clinical features and frequency of abnormal supportive criteria for the diagnosis of CIDP compared to patients fulfilling EFNS/PNS criteria. Two or more abnormal supportive criteria were present in $40(61.2 \%)$ patients raising to $54(80.6 \%)$ if we also included a history of a relapsing course as a possible supportive criteria. Increased cerebrospinal fluid proteins and response to immune therapy most frequently helped in supporting the diagnosis of CIDP. Response to therapy was similarly frequent in patients fulfilling or not EFNS/PNS criteria (87.3\% versus $85.9 \%$ )

Conclusions: Patients with a clinical diagnosis of CIDP had similar clinical findings, frequency of abnormal supportive criteria and response to therapy compared to patients fulfilling EFNS/PNS criteria. The presence of abnormal supportive criteria may help supporting the diagnosis of CIDP in patients with a medical history and clinical signs compatible with this diagnosis but nondiagnostic nerve conduction studies. 


\section{INTRODUCTION}

Chronic inflammatory demyelinating polyradiculoneuropathy (CIDP) is a chronic and often disabling neuropathy with a prevalence ranging from 1 to 9 cases per 100000 [1,2]. The cause of CIDP is still unclear even if several data point to an immune mediated pathogenesis, as also indicated by the frequent improvement of patients after immune therapies [3-6]. The majority of patients with CIDP has a mostly symmetric proximal and distal motor and sensory impairment with decreased or absent deep tendon reflexes and a progressive or relapsing course [3-6]. Several variants have been, however, described based on distribution of symptoms and signs, broadening the spectrum of this disorder [3-7].

Diagnosis of CIDP can be challenging and, in recent years, several different sets of diagnostic criteria have been proposed with variable combinations of electrophysiological and clinical features [8-11]. Currently, the most widely accepted criteria are those recommended by the European Federation of Neurological Societies and Peripheral Nerve Society (EFNS/PNS) [11] that were shown to provide the best combination of sensitivity and specificity (about $75 \%$ and $90 \%$, respectively) for the diagnosis of CIDP compared with the other criteria [12-14]. These criteria allow this diagnosis only in the presence of demyelinating feature in at least one motor nerve. In most reported series, there is indeed a consistent proportion of patients who have the clinical features compatible with a diagnosis of CIDP but who do not fulfill the EFNS/PNS electrodiagnostic criteria [14] and, therefore, might be denied the access to effective therapy. In these patients, a clinical diagnosis of CIDP is often supported by the presence of abnormal ancillary investigations. It is not clear, however, which and how many supportive criteria may help in the diagnosis of these patients, and whether their clinical features and response to therapy are similar to those of the patients who fulfill the EFNS/PNS criteria.

We reviewed the data from patients with a medical history and clinical signs compatible with CIDP and electrodiagnostic criteria not fulfilling the EFNS/PNS electrodiagnostic criteria included in the Italian CIDP database to clarify whether the clinical features, disease course and treatment response was similar to patients fulfilling EFNS/PNS criteria and to identify the relevance of ancillary tests in supporting the diagnosis of CIDP.

\section{PATIENTS AND METHODS}

Database and study population 
From January 2015 to June 2019, we enrolled 582 patients with a clinical diagnosis of CIDP in our web-based database (CINECA, Bologna, Italy). We excluded 24 patients for the presence of a different diagnosis and 23 patients for unavailable neurophysiological data. A total of 535 patients were included in the study. At the time of enrollment, $468(87.5 \%)$ patients fulfilled the EFNS/PNS clinical and electrodiagnostic criteria for CIDP including 430 (92\%) patients with a definite, $33(7 \%)$ with a probable, and three (1\%) with a possible diagnosis of CIDP. We also included among them two patients $(0.4 \%)$ with a typical chronic immune sensory polyradiculopathy (CISP) [15] and normal motor conduction studies. The other 67 (12.5\%) patients had a medical history and clinical signs compatible with the diagnosis of CIDP or one of its variants but did not fulfill the EFNS/PNS electrodiagnostic criteria. We reviewed the data from these patients and compared to those of patients fulfilling these criteria [16]. The Ethic Committee of each participating Center approved the study. All the patients gave written informed consent.

\section{Clinical assessment and ancillary tests}

All patients were subjected to detailed clinical history including time of onset, distribution and progression of symptoms including weakness, sensory symptoms, ataxia, pain, cranial nerve impairment, autonomic dysfunction and the presence of concomitant diseases. Muscle strength was assessed with the Medical Research Council (MRC) scale [17], range 1-60. Neurological disability was evaluated with the INCAT scale [18], range 0-10.

The treating neurologist defined the course of the disease as progressive or relapsing. A relapsing course was defined as a clinical worsening after an initial improvement that was not related to a suspension or reduction of the dose of therapy. However, some patients with a delayed worsening ( $>$ three months) after treatment suspension or reduction might have been also included in this group. An acute onset of CIDP (A-CIDP) was also reported and defined as a neuropathy that was initially diagnosed as Guillain-Barré syndrome (GBS) but that continued to progress or relapse after more than two months from disease onset. The diagnosis of a typical or atypical CIDP phenotype was reviewed in all the patients by the coordinating Center at the time of the inclusion in the study according to our criteria [7]. We defined response to previously performed therapy as a subjective amelioration confirmed by the treating neurologist as an improvement of at least 2 points on the MRC sum score or one point on the INCAT score [19].

Results of cerebrospinal fluid (CSF) examination performed during the course of the disease were reported including total protein level and cell count. As to protein counts, we 
considered as upper reference limit $50 \mathrm{mg} / \mathrm{dl}$ for patients aged $\leq 50$ years and $60 \mathrm{mg} / \mathrm{dl}$ for those aged $>50$ years [20]. The results of brachial/lumbosacral plexus and roots MRI examination were reported and defined by the local examiner of possible supportive value for the diagnosis of CIDP, if they showed an enlargement or T2-hyperintense signal and/or gadolinium enhancement [11]. The results of nerve ultrasound (US) were considered of possible supportive value for the diagnosis of CIDP if the local examiner reported an enlargement of the examined nerves beyond their normal values [21]. The results of nerve biopsy, mostly of the sural nerve, were considered relevant for the diagnosis if the examiner reported signs of demyelination or remyelination by teased fiber analysis or electron microscopy or inflammatory cell infiltrates on paraffin sections.

The results of diagnostic nerve conduction studies (NCS) performed during the course of the disease were included. Motor nerve conduction studies were planned to be performed bilaterally in the median, ulnar, common peroneal and tibial nerves and included distal and proximal (up to the elbow in most patients) compound muscle action potential (CMAP) amplitude (onset to peak) and duration, motor conduction velocities (MCV), distal and proximal motor latencies and in most patients F-wave latency. Sensory conduction studies were planned to be performed bilaterally in the median, ulnar and sural nerves and included sensory action potential (SAP) amplitude, distal latency (DL) and conduction velocity (SCV). There was no definite time point for the examination since each Center was asked to include the most complete and diagnostic examination. Some patients also underwent somatosensory evoked potentials (SSEP) that were considered of diagnostic value if they reflected abnormal conduction velocity in proximal sensory fibers in the absence of signs of central nervous system disease. The reason for suspecting the diagnosis of CIDP beyond the results of nerve conductions studies was also reported in the Database by each center including the abnormality of any supportive criteria and the history of a relapsing course.

All the patients had been extensively investigated in each center for the presence of a possible alternative cause of the neuropathy by clinical and laboratory investigations in accordance with the EFNS/PNS guidelines [11]. Patients with serum IgM monoclonal gammopathy were excluded if they had increased titers of anti-myelin-associated glycoprotein (MAG) IgM antibodies (over 7000 Unit by Buhlman method in our laboratory) [22]. Patients with a concomitant disease including diabetes and monoclonal gammopathy without anti-MAG antibodies were included in the study, as their presence does not exclude the diagnosis of CIDP according to EFNS/PNS criteria. In all the patients we centrally reviewed the clinical features and 
the results of ancillary tests and classified the results of motor and sensory nerve conduction studies according to the EFNS/PNS criteria, to determine the diagnosis of definite, probable or possible CIDP [11].

\section{Statistical analysis}

Descriptive statistics were reported for the sample of patients with CIDP overall and separately for the two subgroups of patients fulfilling or not the EFNS/PNS diagnostic criteria. Categorical variables were described using frequencies and percentages, while continuous variables using mean, medians and ranges. We compared demographic and clinical features, including response to therapy, between different subgroups of patients with the chi-square or the Fisher's exact test for categorical variables, and the t-test or the Wilcoxon-Mann-Whitney test for continuous variables. We performed the analyses with IBM SPSS Statistics for Windows, Version 19.0 (Armonk, NY: IBM Corp, USA).

\section{RESULTS}

\section{Clinical findings and disease course}

The 67 included patients were 45 men (67.2\%) and 22 women (32.8\%) (ratio: 2.0:1), aged 32 to 87 years (mean 60.5; median 62) with a mean age at onset of 52.2 years (median 55; range 15-77 years), a mean disease duration of 8.0 years (median 6 ; range $0.2-37$ years) and a mean INCAT score of 2.4 (median 2; range 0-8). In 49 (73.1\%) patients the clinical phenotype was of typical CIDP and in 18 (26.9\%) of atypical CIDP (Table 1). The progression of the disease was relapsing in 33 patients $(50 \%)$ and progressive in $33(50 \%)$, while in one the data was missing. Two (3.3\%) patients had an acute onset evolving in both cases into a relapsing course. None of the examined demographic and clinical parameters significantly differed from patients with the EFNS/PNS criteria with the only exception of dysphagia or dysphonia that was more frequent in EFNS/PNS patients (Table 1).

\section{Role of supportive criteria}

A similar proportion of patients fulfilling or not the EFNS/PNS electrodiagnostic criteria had increased CSF proteins and comparable levels of the proteins and comparable levels of the proteins were found in the two groups (Table 2). Sensory nerve conduction abnormalities 
consistent with demyelination according to the EFNS/PNS criteria were more frequently found in patients with EFNS/PNS CIDP. Only one patient without EFNS/PNS criteria had delayed SSEP in the lower limbs. This patient also had reduced SCV in the ulnar nerve. Even if they were rarely analyzed in both groups, nerve biopsy findings consistent with demyelination or with inflammatory infiltrates and nerve or roots enlargement or enhancement by MRI or US were similarly frequent in patients fulfilling or not EFNS/PNS criteria. A similar frequent overall response to therapy was also observed between patients fulfilling $(85.9 \%)$ or not $(87.3 \%)$ the EFNS/PNS criteria with a similarly frequent response to IVIg, corticosteroids, plasma exchange or other immune therapies (Table 2).

The presence of abnormal supportive criteria for the diagnosis of CIDP were examined in all patients not fulfilling electrodiagnostic criteria (mean number of supportive criteria examined 2.9 , range 1 to 4 ) with a mean number of abnormal tests of 1.8 (range 1-4). Two or more supportive criteria were found in 41 patients $(61.2 \%)$ while $12(17.9 \%)$ patients had three or more supportive criteria for the diagnosis of CIDP (Figure 1A). When we added the presence of a relapsing course to the supportive criteria, $54(80.6 \%)$ patients had at least two supportive criteria (Figure 1B) including $26(38.8 \%)$ with three of more criteria. Similar figures applied to the 18 patients with an atypical phenotype with 8 patients (55.5\%) having at least two supportive criteria (13 including a relapsing course; $72.2 \%$ ) and two (11.1\%) with three criteria (7 including relapse; $38.9 \%$ ). Since the diagnosis of CIDP should be considered before starting therapy, when we excluded response to therapy from the supportive criteria, two or more supportive criteria were found in $12(17.9 \%)$ raising to $32(47.8 \%)$ if we added the presence of a relapsing course.

\section{Electrodiagnostic studies}

The number of examined motor nerves was lower in patients not fulfilling the EFNS/PNS electrodiagnostic criteria (mean 4.8, median 5, range 2-8) than in EFNS/PNS patients (mean 5.6, median 6 , range $2-8 ; \mathrm{p}<0.0015)$. However, at least four motor nerves were examined in 50 nonEFNS/PNS patients (74.6\%) and six or more nerves in $26(38.8 \%)$ patients. There was no difference between patients with less than four motor nerve examined and those with four or more nerves examined as to the frequency of each abnormal supportive criteria, the proportion of patients with two or more abnormal supportive criteria and the response to therapy. There was no statistically significant difference in the time of executing electrodiagnostic in relation to the onset 
of symptoms between patients fulfilling or not electrodiagnostic criteria (5.1 vs 3.9 years, respectively; $\mathrm{p}>0.05$ ).

We also found some minor, non-diagnostic signs of demyelination in 39 (73.1\%) nonEFNS/PNS patients (14 cases in upper limb nerves, 23 in lower limb nerves and 2 in both). These abnormalities included 30-49\% reduction of proximal-to-distal CMAP amplitude reduction in one nerve excluding the tibial nerve (5 patients); $20-29 \%$ reduction of proximal-to-distal CMAP amplitude in one (5 patients) or more nerves (1 patient) excluding the tibial nerve and the site of nerve compression; 40-49\% proximal-to-distal CMAP amplitude in one tibial nerve (3 patients); $20-29 \%$ reduction of motor conduction velocity in one (11 patients) or two nerves (5 patients) including 10 with normal or less than 20\% distal CMAP amplitude reduction (5 in upper and 5 in lower limb nerves) and 6 with a more pronounced reduction of CMAP amplitude (all in lower limb nerves); F-waves absent in two or more nerves (5 patients) in the absence of other demyelinating features; $40-49 \%$ increased distal latency in one nerve ( 2 patients). In all required cases [10], the distal CMAP amplitude of the negative peak was higher than $20 \%$ of the lower normal limit. The other 28 patients either had minimal sign of possible demyelination (10-20\% reduction of motor conduction velocity or $20-30 \%$ reduction of proximal to distal CMAP amplitude or $30-39 \%$ in tibial nerve, 17 patients) or absence or reduced amplitude distal CMAP (11 patients) in upper ( 3 patients) or lower (22 patients) limbs or both (3 patients). We did not find significant differences between patients with or without these signs beside a higher number of male and higher frequency of abnormal sensory conduction studies in patients having these signs (Supplementary Table).

\section{DISCUSSION}

Since the first formal definition of CIDP by Dyck et al. in 1975 [23], at least 15 diagnostic criteria were proposed with different combinations of clinical, electrophysiological, laboratory, and biopsy features. Different comparison studies confirm that the best combination in terms of sensitivity/specificity [12-14] is offered by the EFNS/PNS criteria [11], which are currently used in most clinical trials in CIDP. A number of supportive investigations were included in these criteria to improve the diagnostic certainty in patients not fulfilling the electrodiagnostic criteria. These investigations support, however, the diagnosis in patients already fulfilling a possible or 
probable diagnosis of CIDP but do not allow this diagnosis in patients not having demyelinating features in at least one motor nerve.

In our series of 535 patients with a diagnosis of CIDP or one of its variants, 468 (87.5\%) patients fulfilled the diagnostic criteria of the EFNS/PNS, while 67 (12.5\%) had a medical history and clinical signs compatible with CIDP with electrodiagnostic studies not fulfilling the EFNS/PNS criteria. None of these patients had clinical or laboratory signs of other possible causes for their neuropathy. These data are in line with the reported sensitivity of these criteria [12-14]. Rajabally and colleagues [14] reported, for instance, that $81.3 \%$ of the patients with CIDP fulfilled the EFNS/PNS criteria for definite or probable CIDP. This percentage is similar to the proportion of our patients with definite or probable CIDP (86.5\%).

Our patients not fulfilling the EFNS/PNS criteria had a similar gender distribution, age at onset, symptoms at onset and during the course of the disease, typical or atypical presentation, disease duration, and INCAT score at enrollment in comparison to EFNS/PNS patients. The progression of the disease was relapsing in about half of the patients in both groups. This figure is higher than in some series [23-24], but similar to others [25-26] possibly reflecting difference in the definition of relapse [4]. It is also possible that some our patients with a delayed worsening after treatment suspension or reduction were deemed to have a relapsing form but this applied for both groups of patients. The only difference between the two groups was a slightly lower frequency of dysphagia or dysphonia in EFNS/PNS patients and a higher frequency of sensory conduction studies consistent with demyelination in this group that is probably consistent with the difference observed in motor nerve conduction studies. There was also no significant difference in the proportion of each abnormal supportive criteria between the two groups. Most importantly, non-EFNS/PNS patients had a similarly frequent overall response to therapy and to each individual therapy compared to EFNS/PNS patients. Even if this data should be considered with caution in a retrospective study, in all our patients the treating neurologist confirmed the subjective amelioration using clinically relevant measures [19].

When we analyzed the factors that might have contributed to the diagnosis of CIDP beside the medical history and clinical presentation, we found that $41(61.2 \%)$ patients had at least two supportive criteria for this diagnosis. This figure raised to $54(80.6 \%)$ if we also considered a relapsing course as a possible supportive criteria for the diagnosis. Even if a relapsing course is part of the clinical definition of CIDP we think that its consideration as possible supportive criterion is justified by its occurrence in only few other neuropathies including vasculitis, acute 
porphyria and episodes of exposure to toxic agents. The distinction with hereditary neuropathy with liability to pressure palsy may be more difficult given the similar presence of signs of demyelination and conduction block. However, the combination of clinical history, presence of other supportive abnormalities, response to therapy and absence of familial history might help in the distinction from these neuropathies. A better definition of relapse in CIDP might be also necessary to uniform the data from different series. The number of supportive criteria in our patients might have been even higher if we consider that nerve US or MRI and nerve biopsy were only performed in a minority of patients to improve the diagnostic definition. This could explain why an invasive test like nerve biopsy was performed in a higher proportion of patients not fulfilling $(16.4 \%)$ than fulfilling $(7.5 \%, \mathrm{p}=0.0321) \mathrm{EFNS} / \mathrm{PNS}$ criteria while non-invasive tests like nerve US or MRI were performed in a similar proportion of patients (12.9\% and $13.8 \%$ ).

One limit of this study is the lower number of examined motor nerve in patients not fulfilling than in those fulfilling the EFNS/PNS electrodiagnostic criteria. Most of non-EFNS/PNS patients (73\%) had, however, four or more motor nerves examined and $38.8 \%$ at least six nerves. Even if there was no difference between patients who had four of more nerves examined or less, it is possible that some patients might have fulfilled the EFNS/PNS electrodiagnostic criteria with a more extensive and complete electrophysiological examination inclusive of a more proximal nerve stimulation in the upper limbs. There was also no difference between patients with or without minor non-diagnostic signs of demyelination indicating that the use of less restrictive electrodiagnostic criteria did not permit to implement the sensitivity of the diagnosis for CIDP.

Even if it is difficult to propose new diagnostic criteria in the absence of a control population, our data suggest that in patients with a medical history and clinical signs compatible with CIDP no other sign of a possible alternative diagnosis and non-diagnostic nerve conduction studies, a diagnosis of possible clinical CIDP might be supported by the presence of two supportive criteria (43.3\% in our series, $40.3 \%$ adding a relapsing course to the criteria), of probable CIDP by three criteria of $(16.4 \%, 29.9 \%$ adding relapse) and of definite clinical CIDP by four criteria $(1.5 \%, 10.4 \%$ adding relapse). We also think that the presence of at least two supportive criteria may also justify the initiation treatment in these patients. This would have allowed initiation of therapy in almost $50 \%$ of our patients if we also consider a history of a relapsing course.

We are aware that even if these criteria may favor the access to the diagnosis and therapy to patients not fulfilling the EFNS/PNS electrodiagnostic criteria, they may also increase the risk 
of over-diagnosis of CIDP, especially in patients with an atypical presentation or with only axonal changes on nerve conduction studies [27-28]. We think, however, that an objective assessment of the medical history and clinical presentation of the patients, the exclusion of other possible causes for the neuropathy and an accurate search for possible supportive criteria for the diagnosis of CIDP might limit this risk and favor the access to treatment of patients that would be otherwise denied a possibly effective therapy.

Limitations of this study include its retrospective nature and the lack of a control population of patients not affected by CIDP. Moreover, US/MRI and nerve biopsy were not routinely performed, so that the percentages of clinical CIDP patients with at least two supportive criteria could be even higher than in our analysis. Data on response to therapy should be also considered with caution considering the retrospective nature of this study. This response was however similar to what reported in the literature [29-34], probably reflecting the fact that the study was performed in centers with expertise in immune mediated neuropathies. Despite these limitations, we think that this study provide the opportunity to verify the usefulness and the critical issues related to the use of current diagnostic criteria for CIDP and supports the opportunity of the revision of these criteria.

Contributors: ENO and GL conceived, organized and designed the study, reviewed and commented on the statistical analysis, wrote the first draft of the report and reviewed the report. FM, DC, RF, CB, MF, LB, GA, GC, SJ, AM, AC, AMC, GAM, AMC, GS, MC, ML, GL, TR, GC, EB, PED, EB, LS, ST, MR, SCP, AS, LL, AT, GM, ES, LG contributed to the conception, organization, and execution of the research project, reviewed and commented on the statistical analysis and the report. DC, FM, GC, MS, PEM, ML and LS, reviewed and discussed the conclusion of the study.

Funding/Support: this study was supported by a Grant from Regione Lombardia, Italy, for patients from this Region and by a Grant of the Italian Ministry of Health 'Ricerca Finalizzata 
2016' (Grant code: RF-2016-02361887) to Humanitas Clinical and Research Center, IRCCS. The study was also supported by unrestricted grants from Kedrion Biopharma (Italy), CSL Behring (Italy), Humanitas Clinical and Research Institute (Milan, Italy), and GBS-CIDP Foundation International (USA). The funding organizations had no role in the design and conduct of the study; collection, management, analysis, and interpretation of the data; preparation, review, or approval of the manuscript; decision to submit the manuscript for publication.

\section{References}

1. Lunn MP, Manji H, Choudhary PP, Hughes RA, Thomas PK. Chronic inflammatory demyelinating polyradiculoneuropathy: a prevalence study in south east England. J Neurol Neurosurg Psychiatry 1999;66:677-680.

2. Laughlin RS, Dyck PJ, Melton LJ III, Leibson C, Ransom J, Dyck PJ. Incidence and prevalence of CIDP and the associations with diabetes mellitus. Neurology 2009;73:39-45.

3. Koller H, Kieseier BC, Jander S, Hartung HP. Chronic inflammatory demyelinating polyneuropathy. N Engl J Med 2005;352:1343-1356.

4. Vallat JM, Sommer C, Magy L. Chronic inflammatory demyelinating polyradiculoneuropathy: diagnostic and therapeutic challenges for a treatable condition. Lancet Neurol 2010;9:402-412.

5. Nobile-Orazio E. Chronic inflammatory demyelinating polyradiculoneuropathy and variants: where we are and where we should go. J Peripher Nerv Syst. 2014;19:2-13.

6. Lehmann HC, Broke N, Kuwabara S. Chronic inflammatory demyelinating polyneuropathy: update on diagnosis, immunopathogenesis and treatment. J Neurol Neurosurg Psychiatry. 2019 Apr 16. pii: jnnp-2019-320314. doi: 10.1136/jnnp-2019-320314. [Epub ahead of print] . 
7. Doneddu PE, Cocito D, Manganelli F, et al. Atypical CIDP: diagnostic criteria, progression, and treatment response. Data from the Italian CIDP Database. J Neurol Neurosurg Psychiatry 2019;90:125-132.

8. Ad Hoc Subcommittee of the American Academy of Neurology AIDS Task Force. Criteria for diagnosis of chronic inflammatory demyelinating polyneuropathy (CIDP). Neurology 1991;41:617-618.

9. Koski CL, Baumgarten M, Magder LS, et al. Derivation and validation of diagnostic criteria for chronic inflammatory demyelinating polyneuropathy. J Neurol Sci 2009;277:1-8.

10. Hughes RA, Bouche P, Cornblath DR, et al. European Federation of Neurological Societies/Peripheral Nerve Society guideline on management of chronic inflammatory demyelinating polyneuropathy: report of a joint task force of the European Federation of Neurological Societies and the Peripheral Nerve Society. Eur J Neurol 2006;13:326-332.

11. Van den Bergh PY, Hadden RD, Bouche P, et al. European Federation of Neurological Societies/Peripheral Nerve Society guideline on management of chronic inflammatory demyelinating polyneuropathy: report of a joint task force of the European Federation of Neurological Societies and the Peripheral Nerve Society-First Revision. Eur J Neurol 2010;17:356-363. Corrigendum in: Eur J Neurol 2011:18:276.

12. Bromberg MB. Review of the evolution of electrodiagnostic criteria for chronic inflammatory demyelinating polyradiculoneuropathy. Muscle Nerve 2011;43:780-794.

13. Breiner A, Brannagan TH III. Comparison of sensitivity and specificity among 15 criteria for chronic inflammatory demyelinating polyneuropathy. Muscle Nerve 2014;50:40-46.

14. Rajabally YA, Nicolas G, Piéret F, Bouche P, Van den Bergh PY. Validity of diagnostic criteria for chronic inflammatory demyelinating polyneuropathy: a multicentre European study. J Neurol Neurosurg Psychiatry 2009;80:1364-1368.

15. Sinnreich M, Klein CJ, Daube JR, et al. Chronic immune sensory polyradiculopathy. A possibly treatable sensory ataxia. Neurology 2004;63:1662-1669.

16. Liberatore G, Manganelli F, Cocito D, et al. Relevance of diagnostic investigations in chronic inflammatory demyelinating polyradiculoneuropathy: data from the Italian CIDP Database. J Peripher Nerv Syst 2020; Apr 28. doi:10.1111/jns.12378 (Epub ahead of print)

17. Kleyweg RP, van der Meché FG, Schmitz PI. Interobserver agreement in the assessment of muscle strength and functional abilities in Guillain-Barré syndrome. Muscle Nerve 1991;14:1103-1109. 
18. Hughes RAC, Bensa S, Willison HJ, et al. Randomized controlled trial of intravenous immunoglobulin versus oral prednisolone in chronic inflammatory demyelinating polyradiculoneuropathy. Ann Neurol 2001;50:195-201.

19. Merkies ISJ, van Nes I, Hanna K, Hughes RAC, Deng C. Confirming the efficacy of intravenous immunoglobulin in CIDP through minimum clinically important differences: shifting from statistical significance to clinical relevance. J Neurol Neurosurg Psychiatry 2010;81:1194-1199.

20. Breiner A, Moher D, Brooks J, Cheng W, Hegen H, Deisenhammer F, et al. Adult CSF total protein upper reference limits should be age partitioned and significantly higher than $0.45 \mathrm{~g} / \mathrm{L}$ : a systematic review. J Neurol 2019;266(3):616-624.

21. Goedee HS, Jongbloed BA, van Asseldonk J-TH, et al. A comparative study of brachial plexus sonography and magnetic resonance imaging in chronic inflammatory demyelinating neuropathy and multifocal motor neuropathy. Eur J Neurol 2017;24:1307-1313.

22. Liberatore G, Giannotta C, Sajeev BP, et al. Sensitivity and specificity of a commercial ELISA test for anti-MAG antibodies in patients with neuropathy. J Neuroimmunol 2020 Aug 15;345:577288. doi: 10.1016/j.jneuroim.2020.577288. Epub 2020 Jun 8.

23. Dyck PJ, Lais AC, Ohta M, Bastron JA, Okazaki H, Groover RV. Chronic inflammatory polyradiculoneuropathy. Mayo Clin Proc 1975;50:621-637.

24. Hattori, N, Misu K, Koike H, Nagamatsu M, Hirayama M, Sobue G. Age of onset influences clinical features of chronic inflammatory demyelinating polyneuropathy, J Neurol Sci 2001;184:57-63.

25. McCombe PA, Pollard JD, McLeod JG. Chronic inflammatory demyelinating polyradiculoneuropathy: a clinical and electrophysiological study on 92 cases. Brain 1987;110:1617-1630.

26. Barohn RJ, Kissel JT, Warmolts JR, Mendell JR. Chronic inflammatory demyelinating polyradiculoneuropathy: clinical characteristics, course and recommendations for diagnostic criteria. Arch Neurol 1989; 46:878-884.

27. Allen JA, Lewis RA. CIDP diagnostic pitfalls and perception of treatment benefit. Neurology 2015;85:498-504.

28. Allen J, Ney J, Lewis RA. Electrodiagnostic errors contribute to chronic inflammatory demyelinating polyneuropathy misdiagnosis. Muscle Nerve 2018;57:542-549. 
29. Cocito D, Paolasso I, Antonini G, et al. A nationwide retrospective analysis on the effect of immune therapies in patients with chronic inflammatory demyelinating polyradiculoneuropathy. Eur J Neurol 2010;17:289-294.

30. Eftimov F, Winer JB, Vermeulen M, de Haan R, van Schaik IN. Intravenous immunoglobulin for chronic inflammatory demyelinating polyradiculoneuropathy. Cochrane Database Syst Rev 2013;12:CD001797.

31. Mehndiratta MM, Hughes RA. Corticosteroids for chronic inflammatory demyelinating polyradiculoneuropathy. Cochrane Database Syst Rev 2012;8:CD002062.

32. Mehndiratta MM, Hughes RA, Agarwal P. Plasma exchange for chronic inflammatory demyelinating polyradiculoneuropathy. Cochrane Database Syst Rev 2012;9:CD003906.

33. Cocito D, Grimaldi S, Paolasso I, et al. Immunosuppressive treatment in refractory chronic inflammatory demyelinating polyradiculoneuropathy. A nationwide retrospective analysis. Eur J Neurol 2011;18:1417-1421.

34. Nobile-Orazio E, Cocito D, Jann S, et al. Intravenous immunoglobulin versus intravenous methylprednisolone for chronic inflammatory demyelinating polyradiculoneuropathy: a randomised controlled trial. Lancet Neurol 2012;11:493-502.

\section{Data availability}

The data that support the findings of this study are available from the corresponding author upon reasonable request. 


\section{LEGENDS:}

Table 1: comparison of demographic and clinical findings in patients with CIDP fulfilling or not EFNS/PNS criteria.

Table 2: comparison of diagnostic and therapeutic findings in patients with CIDP fulfilling or not EFNS/PNS criteria.

Supplementary Table: comparison of demographic, clinical, diagnostic and therapeutic findings in patients with CIDP not fulfilling EFNS/PNS criteria in relation to the presence of minor signs of demyelination

Figure 1: Number of supportive criteria (SC), including (1A) or not including (1B) a relapsing course (R), in 67 patients with clinical CIDP not fulfilling EFNS/PNS electrodiagnostic criteria (numbers in brackets refer to number of patients).

\section{Conflicting Interests}

E.N.O. reported personal fees for Advisory or Scientific Board from Kedrion, Italy, Baxter, Italy, Novartis, Switzerland, CSL-Behring, Italy, LFB, France, Astellas, the Netherlands, outside the submitted work and travel grants to attend Scientific Meeting from Baxter, Grifols, Kedrion, and 
Novartis, Italy. P.E.D. reported travel grants to attend scientific meetings from CSL Behring and Kedrion. G.L. reported travel grants to attend scientific meetings from CSL Behring and Kedrion. D.C. reported honoraria for lecturing from Shire, CSL Behring, and Kedrion and travel grants to attend scientific meeting from Shire, Kedrion, and CSL Behring. E.P. reported travel grants to attend scientific meetings from CSL Behring. R.F. has served on scientific advisory boards for CSL Behring and has received travel grants from Kedrion and CSL Behring to attend scientific meeting. M.C. reported travel grants to attend scientific meetings from Kedrion. A.M. reported travel grants from Kedrion and CSL Behring to attend scientific meeting. C.B. has served on scientific advisory boards for Pfizer and has received travel grants from Kedrion and CSL Behring to attend scientific meeting. G.C. reported travel grants to attend scientific meetings from CSL Behring and Kedrion. B.F. reported travel grants to attend scientific meetings from CSL Behring and liberal contribution from CSL Behring for the neuromuscular diseases centre, outside the submitted work. A.C. reported travel grants to attend scientific meetings from Kedrion. M.L. reported honoraria for scientific board from Pfeizer and Alnylam and travel grants from Pfeizer, Grifols and Kedrion to attend scientific meeting. L.S. reported personal fees for scientific events from CSL Behring and has received travel grants to attend scientific meetings from CSL Behring and Kedrion. F.M. reported personal fees for scientific events from CSL Behring and has received travel grants to attend scientific meetings from CSL Behring and Kedrion. G.C. reported honoraria for lecturing and travel grants to attend scientific meetings from Kedrion. M.F. has served on scientific advisory boards for CSL Behring and Sarepta Therapeutics and has received travel grants from Sanofi Genzyme, Kedrion, Baxter and CSL Behring to attend scientific meeting. S.J. reported research grants from Grifols, outside this work, and travel grants from Grifols and Kedrion. G.A.M. reported consulence fees and travel fundings from CSL Behring, Kedrion, Shire and Grifols. G.A. reported honoraria for lecturing from Kedrion and Sanofi-genzyme, travel grants from Kedrion, Sanofi-Genzyme and LJ Pharma. G.M. reported consulence fees and travel fundings from CSL Behring, Kedrion, Shire and Grifols. The other authors declare no conflict of interest.

\section{Italian CIDP Database study group}

Pietro Emiliano Doneddu, Giuseppe Liberatore, Francesca Gallia, and Eduardo Nobile-Orazio from the Department of Medical Biotechnology and Translational Medicine, Neuromuscular and Neuroimmunology Service, Humanitas Clinical and Research Institute, Milan University, 
Rozzano, Milan, Italy; Erdita Peci and Dario Cocito from the Department of Neuroscience, University of Turin, Turin, Italy. Stefano Tronci and Raffaella Fazio from the Division of Neuroscience, Department of Neurology, Institute of Experimental Neurology (INSPE), San Raffaele Scientific Institute, Milan, Italy; Fiore Manganelli, Lucio Santoro and Emanuele Spina from the Department of Neuroscience, Reproductive Sciences and Odontostomatology, University of Naples 'Federico II', Naples, Italy; Marta Ruiz and Chiara Briani from the Neurology Unit, Department of Neuroscience, University of Padua, Padua, Italy. Stefano Cotti Piccinelli and Massimiliano Filosto from the Center for Neuromuscular Diseases and Neuropathies, Unit of Neurology ASST 'Spedali Civili', University of Brescia, Brescia, Italy; Alessandro Beronio and Luana Benedetti from the Neurology Unit, Sant'Andrea Hospital, La Spezia, Italy; Antonio Toscano, Luca Gentile and Anna Mazzeo from the Department of Clinical and Experimental Medicine, Unit of Neurology, University of Messina, Messina, Italy; Giorgia Mataluni and Girolama Alessandra Marfia from the Disimmune Neuropathies Unit, Department of Systems Medicine, Tor Vergata University of Rome, Rome, Italy; Laura Piccolo, and Andrea Cortese from the IRCCS Foundation C. Mondino National Neurological Institute, Pavia, Italy; Giuseppe Cosentino and Brigida Fierro from the Department of Experimental BioMedicine and Clinical Neurosciences (BioNeC), University of Palermo, Palermo, Italy; Verrengia Elena Pinuccia and Stefano Jann from the Department of Neuroscience, Niguarda Ca' Granda Hospital, Milan, Italy; Elisa Bianchi and Ettore Beghi from the Laboratorio di Malattie Neurologiche, IRCCS-Istituto Mario Negri, Milan, Italy; Angelo Maurizio Clerici from the Neurology Unit, Circolo \& Macchi Foundation Hospital, Insubria University, DBSV, Varese, Italy; Federica Scrascia and Marinella Carpo from the ASST Bergamo Ovest-Ospedale Treviglio, Treviglio, Italy; Martina Garnero and Angelo Schenone from the Department of Neuroscience, Rehabilitation, Ophthalmology, Genetics, Maternal and Child Health, University of Genoa and IRCCS AOU San Martino-IST, Genoa, Italy; Marco Luigetti and Mario Sabatelli from the Department of Neurology, Catholic University of Sacred Heart, Rome, Italy; Patrizia Dacci and Giuseppe Lauria from the Unit of Neuroalgology, IRCCS Foundation "Carlo Besta" Neurological Institute, Milan, Italy; Luca Leonardi and Giovanni Antonini from the Unit of Neuromuscular Diseases, Department of Neurology Mental Health and Sensory Organs (NESMOS), Faculty of Medicine and Psychology, 'Sapienza' University of Rome, Sant'Andrea Hospital, Rome, Italy; Tiziana Rosso from the Azienda UL.SS. 8 Asolo, Castelfranco Veneto, Italy; Erika Schirinzi and Gabriele Siciliano from the Neurology Unit, Department of Clinical and Experimental Medicine, University of Pisa, Pisa, 
Italy; Claudia Balducci and Guido Cavaletti from the School of Medicine and Surgery and Experimental Neurology Unit, University of Milano-Bicocca, Monza, Italy.

This article is protected by copyright. All rights reserved 
Table 1: comparison of demographic and clinical findings in patients with CIDP fulfilling or not EFNS/PNS criteria.

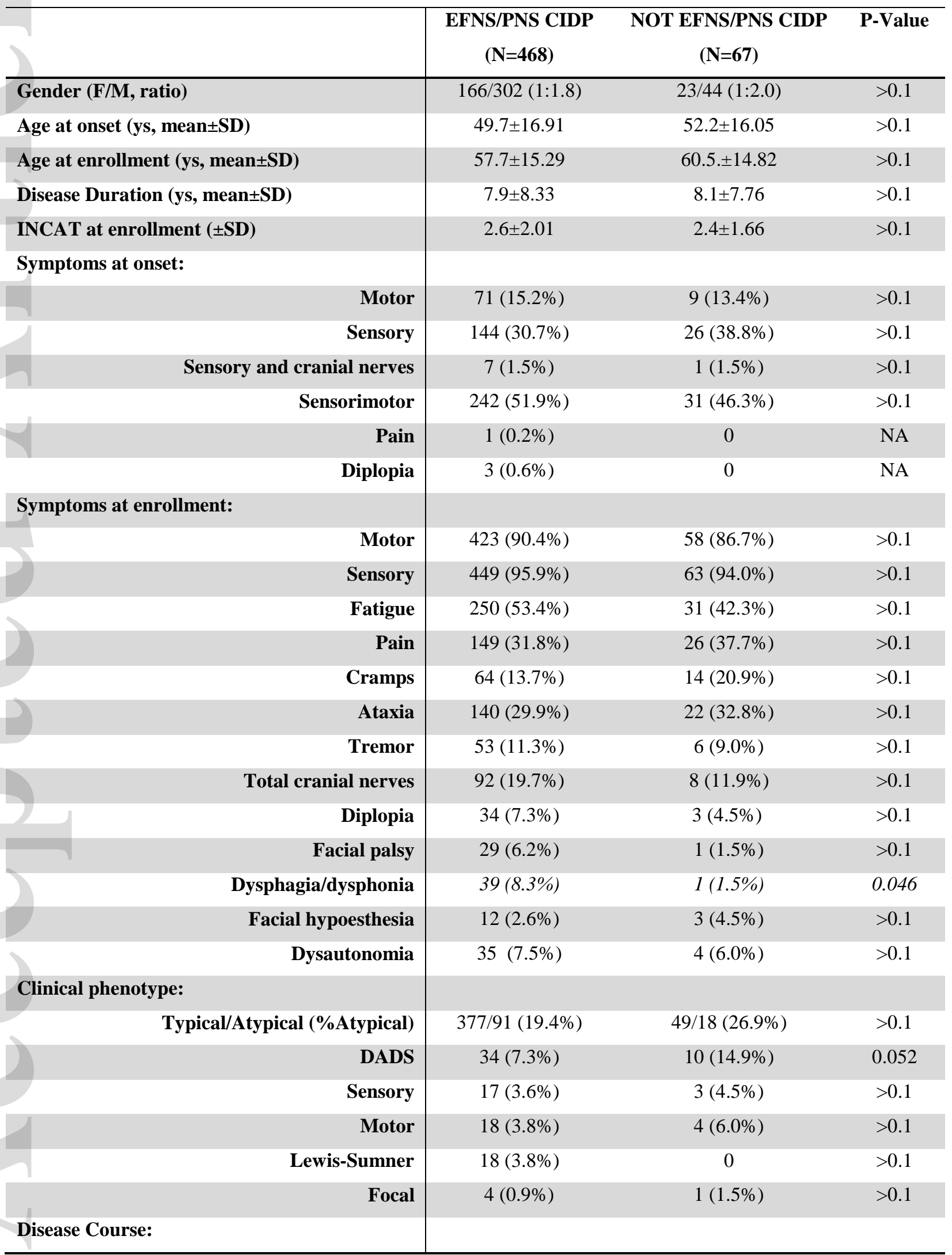

This article is protected by copyright. All rights reserved 


\begin{tabular}{l|ccc}
\hline \multicolumn{1}{|c|}{ Progressive/Relapsing (\% Relapsing) } & $212 / 252(54.3 \%)$ & $33 / 33(50.0 \%)$ & $>0.1$ \\
\multicolumn{1}{|c|}{ Acute Onset } & $43(9.2 \%)$ & $2(3.0 \%)$ & $>0.1$ \\
$\begin{array}{l}\text { Concomitant diseases: } \\
\text { Diabetes } \\
\text { Monoclonal gammopathy (not anti-MAG) }\end{array}$ & $59(12.6 \%)$ & $3(4.5 \%)$ & 0.064 \\
\hline
\end{tabular}

This article is protected by copyright. All rights reserved 
Table 2: comparison of diagnostic and therapeutic findings in patients with CIDP fulfilling or not EFNS/PNS criteria. CV: conduction velocity. SSEP: somato-sensory evoked potential.

\begin{tabular}{|c|c|c|c|}
\hline & $\begin{array}{c}\text { EFNS/PNS CIDP } \\
(n=468)\end{array}$ & $\begin{array}{l}\text { NOT EFNS/PNS CIDP } \\
\qquad(\mathrm{n}=67)\end{array}$ & P-Value \\
\hline \multicolumn{4}{|l|}{ Supportive criteria: } \\
\hline Increased CSF proteins/tested & $280 / 358(78.2 \%)$ & $41 / 50(82.0 \%)$ & $>0.1$ \\
\hline mean $(\mathrm{mg} / \mathrm{dl})$ & 103.3 & 97.2 & $>0.1$ \\
\hline median (mg/dl) & 77 & 75 & $>0.1$ \\
\hline Sensory nerve demyelination/tested & $148 / 423(35.0 \%)$ & $13 / 61(23.2 \%)$ & 0.041 \\
\hline Sensory CV/med abnormal-sur norm. & $120 / 18$ & $12 / 1$ & $>0.1$ \\
\hline Delayed SEP (+ other sensory imp.) & $10(+4)$ & $0(+1)$ & $>0.1$ \\
\hline $\begin{array}{l}\text { Demyelination-Inflammation at nerve } \\
\text { biopsy/tested }\end{array}$ & $20 / 35(57.1 \%)$ & $7 / 11(63.6 \%)$ & $>0.1$ \\
\hline US-MRI abnormalities/tested & $51 / 64(79.7 \%)$ & $7 / 9(77.8 \%)$ & $>0.1$ \\
\hline Response to overall therapies/treated & $366 / 426(85.9 \%)$ & $53 / 63(84.1 \%)$ & $>0.1$ \\
\hline Response to IVIg/treated & $265 / 360(73.6 \%)$ & $38 / 52(73.1 \%)$ & $>0.1$ \\
\hline Response to steroids/treated & $134 / 249(53.8 \%)$ & $21 / 42(50.0 \%)$ & $>0.1$ \\
\hline Response to Plasma exchange/treated & $25 / 43(58.1 \%)$ & $2 / 6(33.3 \%)$ & $>0.1$ \\
\hline Response to Immunosuppressants/treated & $29 / 77(37.7 \%)$ & $3 / 10(30.0 \%)$ & $>0.1$ \\
\hline
\end{tabular}

This article is protected by copyright. All rights reserved 

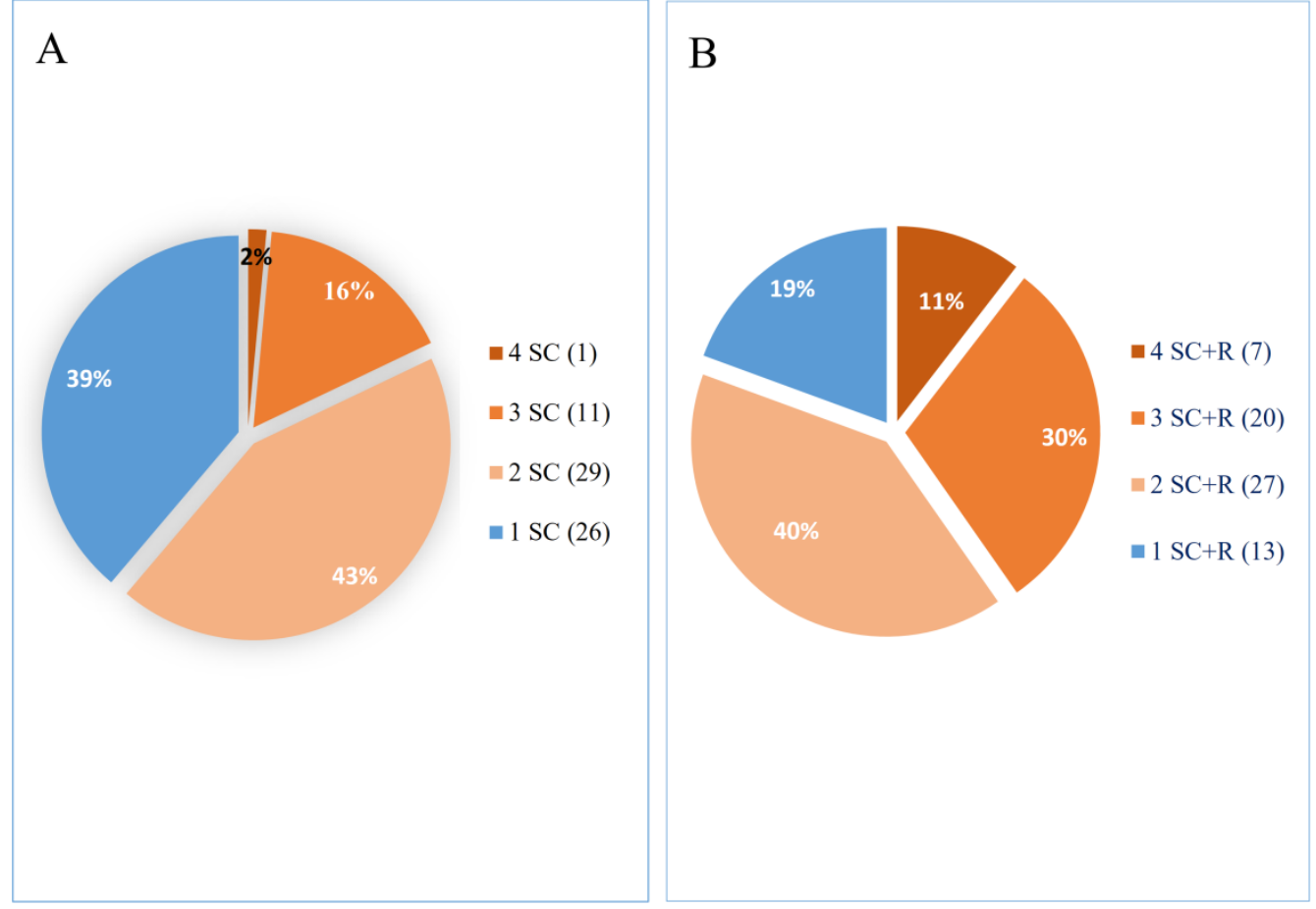

ene_14545_f1.tif

This article is protected by copyright. All rights reserved 


\section{More than 20 years of research in the field of Parkinson's}

Our mission is to empower people with Parkinson's Helping improve the quality of life of People with

\section{Bg⿵冂𠃍} parkinson's PD since 2016

Empowering people with Parkinson's

- Positive online community around Parkinson's Disease

- Find relevant and useful content for PWP, caregivers and HCP's

- Content to help Keeping life ON

- To generate awareness about the complexity of Parkinson's

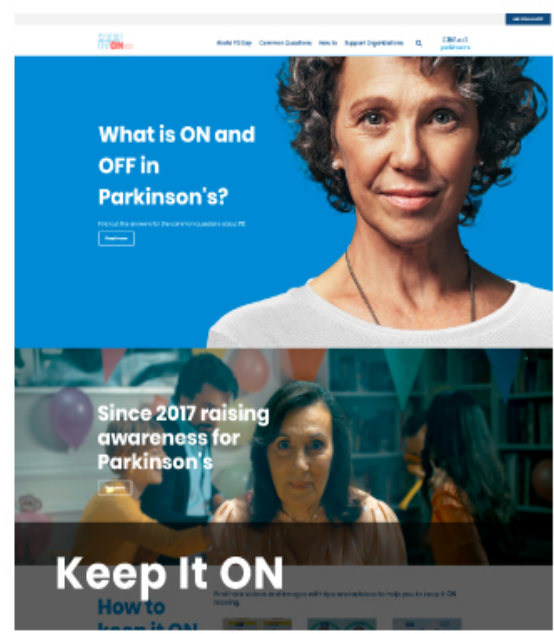

Know More

HCP-exclusive content about Parkinson's, disease management, COMT inhibitors and Bial solutions for PD

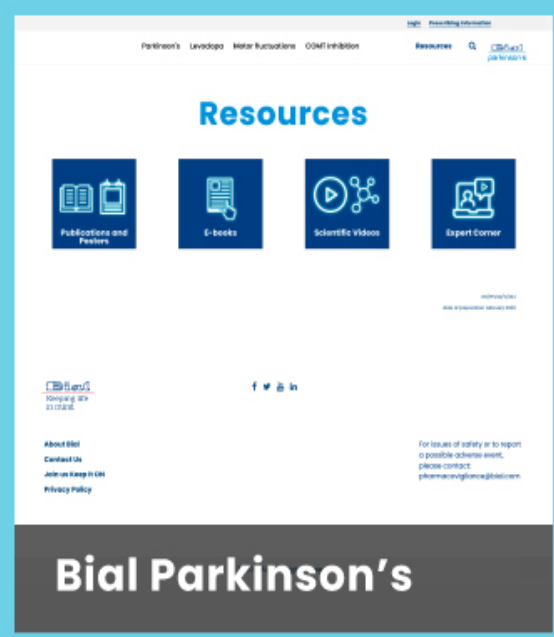

Know More

Bial Parkinson's 